\title{
Isgur-Wise functions from the MIT bag model *
}

\author{
M. SADZIKOWSKI ${ }^{\dagger}$ \\ Institute of Physics, Jagellonian University, \\ ul. Reymonta 4, PL-30059 Kraków, Poland. \\ and \\ K. ZALEWSKI ${ }^{\text {霖 }}$ \\ Institute of Physics, Jagellonian University, \\ ul. Reymonta 4, PL-30059 Kraków, Poland.
}

\begin{abstract}
The Isgur-Wise functions for the ground state to ground state semileptonic decays involving $b \rightarrow c$ transitions are calculated from the (modified) MIT bag model. It is checked that the results for the decays $\bar{B} \rightarrow D l \bar{\nu}$ and $\bar{B} \rightarrow D^{*} l \bar{\nu}$ agree well with experiment. Predictions for the decays $\Lambda_{b} \rightarrow \Lambda_{c} l \bar{\nu}, \bar{B}_{s} \rightarrow D_{s} l \bar{\nu}$ and $\bar{B}_{s} \rightarrow D_{s}^{*} l \bar{\nu}$ are given and discussed.
\end{abstract}

\section{Introduction}

According to the heavy quark paradigm a hadron containing one heavy quark consists of the heavy quark almost immobile at the centre of the hadron

\footnotetext{
*Work supported in part by the KBN grants no. 20-38-09101.

${ }^{\dagger}$ E-mail UFSADZIK@PLKRCY11.BITNET

‡E-mail ZALEWSKI@VSK01.IFJ.EDU.PL

$\S$ also Institut of Nuclear Physics, Kraków
} 
and of a light component surrounding it. In general the light component is very complicated. In the valence quark approximation, however, the light component of a meson is a light (anti)quark and the light component of a baryon is a light diquark. This picture, familiar from the nonrelativistic quark model, cf. e.g. [1], can be derived in a model independent way from QCD, when the mass of the heavy quark $m_{Q}$ tends to infinity [2]. Therefore, each good model retaining its validity in the limit $m_{Q} \rightarrow \infty$ should be possible to interpret as the heavy quark picture modified by some finite mass corrections.

One of us (M.S.) extending and updating ealier work [3], [4], [5] has discussed from this point of view the predictions of a suitably modified MIT bag model for the mass spectra of mesons and baryons containing heavy quarks [6]. In the present paper we discuss some semileptonic decays, where the $b$ quark goes over into a $c$ quark and an $l \bar{\nu}$ pair. Our main result is that the bag model gives very reasonable Isgur-Wise functions. For the decays $\bar{B} \rightarrow D l \bar{\nu}$ and $\bar{B} \rightarrow D^{*} l \bar{\nu}$ (in the following denoted together $\bar{B} \rightarrow D^{(*)} l \bar{\nu}$ ) these results can be directly compared with experiment and the agreement is very satisfactory. We also give predictions for the related decay $\bar{B}_{s} \rightarrow D_{s}^{(*)} l \bar{\nu}$. For the decays $\Lambda_{b} \rightarrow \Lambda_{c} l \bar{\nu}$ we find that the Isgur-Wise function is much steeper than for $B$ decays and consequently we predict that the fraction of the decays $\Lambda_{b} \rightarrow X_{c} l \bar{\nu}$, where $X_{s}=\Lambda_{c}$ is smaller than the corresponding fraction for the decays $\bar{B} \rightarrow D^{(*)} l \bar{\nu}$ in spite of kinematical factors working in the opposite direction. Moreover, we derive a new, restrictive and almost model-independent lower bound for the parameters $\rho^{2}$ related to the IsgurWise functions. Finally, we comment on the implications of our analysis for rare decays like $B \rightarrow K^{*} \gamma$.

\section{Decays $H_{b} \rightarrow H_{c} l \bar{\nu}$ in the heavy quark limit}

The standard formula [7] for the width of the decay of hadron $H_{b}$ into hadron $H_{c}$ and an $l \bar{\nu}$ pair can be written in the form

$$
\frac{d \Gamma}{d q^{2}}=\frac{\left|\vec{p}_{H c}\right|}{128 \pi^{3} m_{H b}^{2}} \overline{|\mathcal{M}|^{2}}
$$

The momentum of $H_{c}$ is calculated in the $H_{b}$ rest frame and the square of the absolute value of the invariant amplitude $\mathcal{M}$ is averaged over the angular distribution of $H_{c}$ in the $H_{b}$ rest frame and of the lepton in the $l \bar{\nu}$ rest frame. 
We define $q^{\mu}=p_{l}^{\mu}+p_{\bar{\nu}}^{\mu}$ so that the $l \bar{\nu}$ rest frame can be referred to as the $\vec{q}=0$ frame. The invariant amplitude can be written in the form

$$
\mathcal{M}=\frac{G_{F} V_{c b}}{\sqrt{2}} \bar{u}_{e} \gamma^{\mu}\left(1-\gamma^{5}\right) v_{\nu}<H_{c}\left|J_{\mu}\right| H_{b}>
$$

We choose the $z$-axis in the $\vec{q}=0$ frame along the momentum of $H_{b}$ as seen in this frame. In the $\vec{q}=0$ frame, neglecting the masses of the leptons, the time-component of the leptonic current vanishes and the space components are

$$
2 \bar{u}_{e} \vec{\gamma} v_{\nu}=-2 \sqrt{q^{2}}\left(i \sin \phi_{l}+\cos \theta_{l} \cos \phi_{l},-i \cos \phi_{l}+\cos \theta_{l} \sin \phi_{l},-\sin \theta_{l}\right)
$$

In the heavy quark limit (further quoted HQET) the hadron current can be expressed by the quark current and the Isgur-Wise functions. The formula [8] reads

$$
\begin{array}{r}
<H_{c}\left|J_{\mu}\right| H_{b}>=\sum_{\lambda}<J_{H b l}, \lambda ; \frac{1}{2}, \lambda_{b} \mid J_{H b}, \lambda_{H b}> \\
<J_{H c l}, \lambda ; \frac{1}{2}, \lambda_{c} \mid J_{H c}, \lambda_{H c}>\sqrt{\frac{\omega+1}{2}} \bar{u}_{c} \gamma_{\mu}\left(1-\gamma^{5}\right) u_{b} \xi_{\lambda}(\omega)
\end{array}
$$

Here $\langle\ldots| \ldots>$ are ordinary Clebsch Gordan coefficients, $J_{H Q l}$ is the angular momentum of the light component of hadron $H_{Q}$ in the $H_{Q}$ rest frame and $\lambda$ is its (conserved) projection on the $z$-axis chosen in the $H_{b}$ rest frame along the direction of the $H_{c}$ momentum. The Isgur-Wise functions $\xi_{\lambda}(\omega)$ are, except for a trivial kinematical factor, overlap functions of the light component of $H_{b}$ at rest with the light component of $H_{c}$ moving with the Lorentz factor $\omega$ in the $+z$ direction

$$
\xi_{\lambda}(\omega)=\sqrt{\frac{2}{\omega+1}}<\Phi_{H c l} \mid \Phi_{H b l}>
$$

Since the Isgur-Wise function depends only on the light components, it does not depend on the spin $J_{H Q}$. For instance, it is the same for both decays $\bar{B} \rightarrow D^{(*)} l \bar{\nu}$.

The number of independent Isgur-Wise functions for a given decay is reduced by the identity 8 


$$
\xi_{\lambda}(\omega)=\eta_{H b} \eta_{H c}(-1)^{J_{H c}-J_{H b}} \xi_{-\lambda}(\omega)
$$

where $\eta_{H Q}$ is the internal parity of the light component of hadron $H_{Q}$.

The quark current in the $H_{b}$ rest frame is

$$
\bar{u}_{c} \gamma^{\mu}\left(1-\gamma^{5}\right) u_{b}=\sqrt{m_{b} m_{c}} \sqrt{\frac{2}{\omega+1}}\left(\omega+1-2 \lambda_{c} v_{c}\right) \phi_{c}^{\dagger} \tilde{\sigma}^{\mu} \phi_{b}
$$

In this formula: $v_{c}=\left|\vec{v}_{c}\right|$. Here and in the following by velocity we always mean the fourvector velocity $v^{\mu}=p^{\mu} / m$. The quark spinors are $\phi_{i}=\left(\begin{array}{l}1 \\ 0\end{array}\right)$ for helicity $+1 / 2$ and $\phi_{i}=\left(\begin{array}{l}0 \\ 1\end{array}\right)$ for helicty $-1 / 2$. The object $\tilde{\sigma}^{\mu}=(1,-\vec{\sigma})$. Substituting this current into formula (4) and contracting the result with the lepton current transformed to the $H_{b}$ rest frame:

$$
\begin{aligned}
\bar{u}_{l} \gamma^{\mu}\left(1-\gamma^{5}\right) v_{\nu}= & 2\left(q_{z} \sin \theta_{l}, \sqrt{q^{2}}\left(i \sin \phi_{l}+\cos \theta_{l} \cos \phi_{l}\right),\right. \\
& \left.\sqrt{q^{2}}\left(-i \cos \phi_{l}+\cos \theta_{l} \sin \phi_{l}\right), q_{0} \sin \theta_{l}\right)
\end{aligned}
$$

one finds from (2) the invariant amplitude and from (11) the decay width.

For further reference we quote the results for the decay $\bar{B} \rightarrow D l \bar{\nu}$ :

$$
\frac{d \Gamma}{d q^{2}}=\frac{G_{F}^{2}\left|V_{c b}\right|^{2}\left|\vec{p}_{D}\right|^{3}}{24 \pi^{3}} \frac{\left(m_{B}+m_{D}\right)^{2}}{4 m_{B} m_{D}} \xi_{\frac{1}{2}}^{2}(\omega)
$$

for $\bar{B} \rightarrow D^{*} l \bar{\nu}$ (the subscripts of $\Gamma$ refer to the helicities of $D^{*}$ ):

$$
\begin{aligned}
\frac{d \Gamma_{ \pm}}{d q^{2}} & =\frac{G_{F}^{2}\left|V_{c b}\right|^{2}\left|\vec{p}_{D^{*}}\right|}{48 \pi^{3}} \frac{m_{D^{*}}}{m_{B}} q^{2}\left(\omega \mp v_{D^{*}}\right)(\omega+1) \xi_{\frac{1}{2}}^{2}(\omega) \\
\frac{d \Gamma_{0}}{d q^{2}} & =\frac{G_{F}^{2}\left|V_{c b}\right|^{2}\left|\vec{p}_{D^{*}}\right|}{96 \pi^{3}} \frac{m_{D^{*}}}{m_{B}}\left(m_{B}-m_{D^{*}}\right)^{2}(\omega+1)^{2} \xi_{\frac{1}{2}}^{2}(\omega)
\end{aligned}
$$

and for $\Lambda_{b} \rightarrow \Lambda_{c} l \bar{\nu}$ (averaged over the initial helicity):

$$
\frac{d \Gamma}{d q^{2}}=\frac{G_{F}^{2}\left|V_{c b}\right|^{2}\left|\vec{p}_{\Lambda_{c}}\right|}{48 \pi^{3}} \frac{m_{\Lambda_{c}}}{m_{\Lambda_{b}}}\left[3 \omega q^{2}+2 m_{\Lambda_{c}} m_{\Lambda_{b}}\left(\omega^{2}-1\right)\right](\omega+1) \bar{\xi}_{\frac{1}{2}}^{2}(\omega)
$$


The bar over $\xi$ in the last formula serves as a remainder that this Isgur-Wise function is different from that in the preceding formulae. For the corresponding decays involving $\bar{b} \rightarrow \bar{c}$ transitions the only modification in the formulae is that the sign of $v_{c}$ should be changed.

According to custom, we have put everywhere particle masses for the quark masses. The difference being of order $O\left(m_{Q}^{0}\right)$ is formally negligible at leading order of HQET, but numerically it may be quite important. With obvious changes these formulae can be also applied to related decays like $\bar{B}_{s} \rightarrow D_{s}^{(*)} l \bar{\nu}$.

It is seen that the only unknown factors in these formulae are the IsgurWise functions $\xi$. Even they drop out from expressions for ratios of decay widths of a given decay into various helicity states at given $q^{2}$. For instance for the decay $\bar{B} \rightarrow D^{*} l \bar{\nu}$ the polarization coefficient $\alpha$ is related to

$$
\left(\frac{d \Gamma_{+}}{d q^{2}}+\frac{d \Gamma_{-}}{d q^{2}}\right) / 2 \frac{d \Gamma_{0}}{d q^{2}}=\frac{2 \omega}{\omega+1} \frac{q^{2}}{q_{\max }^{2}}
$$

where the maximum square of the momentum transer $q_{\max }^{2}=\left(m_{B}-m_{D^{*}}\right)^{2}$, while the asymmetry parameter $A_{f b}$ is

$$
-\frac{3}{4}\left(\frac{d \Gamma_{+}}{d q^{2}}-\frac{d \Gamma_{-}}{d q^{2}}\right) /\left(\frac{d \Gamma_{+}}{d q^{2}}+\frac{d \Gamma_{0}}{d q^{2}}+\frac{d \Gamma_{-}}{d q^{2}}\right)=\frac{3 q^{2}}{4 q^{2} v_{D^{*}}+(\omega+1) q_{\max }^{2}} v_{D^{*}}
$$

In practice, however, in order to compare these formulae with experiment one has to average over $q^{2}$ with weights taking into account the experimenal cut offs. This again requires a knowledge of the Isgur-Wise functions.

\section{$3 \quad$ The Isgur-Wise functions}

The calculation of the Isgur-Wise functions $\xi_{\lambda}(\omega)$ reduces to the calculation of the overlaps $<\Phi_{H c l} \mid \Phi_{H b l}>$. In the present paper we consider only the so-called ground state to ground state transitions, where $\xi(1)=1$ 】. Starting from a model reliable for small recoil velocities, one can either argue that the recoils occuring in the semileptonic $b \rightarrow c$ decays are small (cf. e.g. [9]), or determine the parameter $\rho^{2}$ in the expansion

\footnotetext{
${ }^{1}$ In the following we suppress the subscript $\lambda$, since this leads to no confusion
} 


$$
\xi(\omega)=1-\rho^{2}(\omega-1)+O\left((\omega-1)^{2}\right)
$$

and use some plausible functional form to continue $\xi(\omega)$ (cf. e.g. [1]). A formula particularly convenient for calculations is [1]

$$
\xi(\omega)=\exp \left[-\rho^{2}(\omega-1)\right]
$$

but many other formulae have been suggested [10], [11], [12], [13], [14], [15], [16]. The values of $\rho^{2}$ proposed in the literature range from $\frac{1}{3}$ [16] to infinity [12]. For given data the best $\rho^{2}$ depends strongly on the functional form chosen for $\xi(\omega)$. Thus e.g. ARGUS from its data finds [17] values from 1.17 to 2.31 depending on the choice of the function.

We discuss the Isgur-Wise functions from the point of view of the (modified) MIT bag model [6], but some of our conclusions are more general than the model. Let us note first that the overlap should be a decreasing function of $\omega$. Thus

$$
\xi(\omega) \leq \sqrt{\frac{2}{\omega+1}}=1-\frac{\omega-1}{4}+O\left((\omega-1)^{2}\right)
$$

This bound has been pointed out by de Rafael and Taron [14]. The implication that $\rho^{2}>\frac{1}{4}$ is known as the Bjorken limit [18].

The calculations in the framework of the MIT bag model depend on the reference frame chosen. We choose the (modified [19]) Breit frame, where the velocities of $H_{b}$ and $H_{c}$ are equal and opposite. The velocity of $H_{c}$ will be denoted $\vec{v}$ and its Lorentz factor $\gamma$. Let us note the kinematical identities

$$
\gamma=\sqrt{1+\vec{v}^{2}}=\sqrt{\frac{\omega+1}{2}}
$$

In the Breit frame the overlap function for mesons has the form

$$
<\Phi_{H c l} \mid \Phi_{H b l}>=\int_{C B} \Phi^{\dagger}\left(L_{\vec{v}}^{-1}(0, \vec{x})\right) S^{\dagger}(\vec{v}) S(-\vec{v}) \Phi\left(L_{-\vec{v}}^{-1}(0, \vec{x})\right) d^{3} x
$$

Here

$$
\Phi(t, \vec{x})=\Phi(\vec{x}) \exp \left(-i E_{q} t\right)
$$


is the wave function of the light component in its rest frame and $E_{q}$ is the energy of the light quark. For the ground state to ground state transitions, which we are considering, these functions for $H_{b}$ and $H_{c}$ are identical, thus we omit the subscripts. Choosing the $z$-axis along the vector $\vec{v}$, we have the Lorentz transformations

$$
L_{ \pm \vec{v}}^{-1}(0, \vec{x})=(\mp v z, x, y, \gamma z)
$$

The wave functions relevant for the overlap integral are at $t=0$ in the Breit frame and not at $t=0$ in the rest frames of the particles. For $\vec{v} \neq 0$ this will introduce oscillating factors into the integrand and reduce the overlap. The factors $S( \pm \vec{v})$ are Lorentz boosts. We only need to know that they are real. Thus $S^{\dagger}(\vec{v})=S(\vec{v})=S^{-1}(-\vec{v})$ and the two boost factors cancel. In the calculation of the normalization integral for a bag moving with velocity $\vec{v}$, the corresponding factor $S^{\dagger}(\vec{v}) S(\vec{v}) \neq 1$ cancels the effect of the Lorentz contraction of the bag. The absence of this term gives a further reduction of the overlap for $\omega \neq 1$. The integration is over the Lorentz - contracted bag $\mathrm{CB}$, which is an ellipsoid with semiaxes of length $R$ in the directions perpendicular to the $z$-axis and of length $R / \gamma$ along the $z$-axis. Rescaling the $z$-variable by a factor $\gamma$ we may rewrite the overlap as an integral over a spherical bag $B$ of radius $R$

$$
<\Phi_{H c l} \mid \Phi_{H b l}>=\gamma^{-1} \int_{B} \Phi^{\dagger}(\vec{x}) \Phi(\vec{x}) e^{2 i v \gamma^{-1} E_{q} z} d^{3} x
$$

A baryon differs from the meson in that it has two light quarks in the light componenet. Therefore, its overlap function is just the square of the overlap (22).

Since the integral in (22) is a decreasing function of $v$, we find the inequality

$$
\xi(\omega) \leq \sqrt{\frac{2}{\omega+1}}^{N+1}=1-\frac{1+N}{4}(\omega-1)+O\left((\omega-1)^{2}\right)
$$

where $N=1$ for mesons and $N=2$ for baryons. The corresponding limits on $\rho^{2}$

$$
\rho^{2} \geq \frac{1+N}{4}
$$


are already of practical interest. E.g. Blok and Shifman 20 from a particularly careful analysis of sum rules obtain for the $B$ meson $\rho^{2}=0.65 \pm 0.15$, just above our limit.

In order to calculate $\rho^{2}$ from the full formula for the overlap, it is enough to consider terms up to second order in velocity. Using the spherical symmetry of the ground state one obtains

$$
\rho^{2}=\frac{N+1}{4}+\frac{1}{3} N E_{q}^{2}<r^{2}>
$$

In the bag model the product $E_{q}^{2}<r^{2}>$ is a slowly increasing function of the dimensionless variable $m_{q} R$. We show it in Fig. 1 . Using the model it is possible to express the bag radius $R$ by the masses of the quarks constituting the light component. The resulting dependence of $\rho^{2}$ on the quark mass(es) is shown in Fig. 2 for three cases: for mesons the variable is the mass of the light quark, for barions with two light quarks of equal mass like $\Lambda_{c}$ or $\Omega_{c}$ the variable is the mass of either quark, for baryons with one zero mass quark and one strange quark the variable is the mass of the strange quark. Using the figure it is possible to find the bag model prediction for $\rho^{2}$ corresponding to the ground state of any particle with exactly one heavy quark. For the decays $\bar{B} \rightarrow D^{(*)} l \bar{\nu}$ and $\Lambda_{b} \rightarrow \Lambda_{c} l \bar{\nu}$ we have $m_{q}=0$, which corresponds to

$$
\begin{aligned}
\rho_{B}^{2} & =1.239 \\
\rho_{\Lambda}^{2} & =2.228
\end{aligned}
$$

For the decays $\bar{B}_{s} \rightarrow D_{s}^{(*)} l \bar{\nu}$ we use [6] $m_{s}=0.291$, which yields

$$
\rho_{B_{s}}^{2}=1.625
$$

An exact evaluation of the overlap integral for finite $\omega-1$ yields a result, which is very well approximated by the function deduced from a study of sum rules by Neubert $13 \|$. The error of this approximation for $1 \leq \omega \leq 3$ is less than 1 per cent. Thus, we use for $\bar{B} \rightarrow D^{(*)} l \bar{\nu}$

$$
\xi^{(B)}(\omega)=\left(\frac{2}{\omega+1}\right)^{2+\frac{0.6}{\omega}}
$$

and consequently for $\Lambda_{b} \rightarrow \Lambda_{c} l \bar{\nu}$ 


$$
\xi^{\Lambda}(\omega)=\left(\frac{2}{\omega+1}\right)^{3.5+\frac{1.2}{\omega}}
$$

For $\bar{B}_{s} \rightarrow D_{s}^{(*)} l \bar{\nu}$ we find similarly

$$
\xi^{B_{s}}(\omega)=\left(\frac{2}{\omega+1}\right)^{2.7+\frac{0.6}{\omega}}
$$

with an error below two per cent for $1 \leq \omega \leq 3$.

A comparison of our function $\xi^{B}(\omega)$ with the recent ARGUS data is show in Fig. 3. As a by-product of this comparison we obtain the element of the Cabibbo-Kobayashi-Masakawa matrix: $V_{c b}=0.0420 \sqrt{\left(\tau_{B} / 1.29 p s\right)}$. The $\chi^{2}$ of this fit is 7.0 for 7 degrees of freedom, which is very satisfactory. As a further check we have calculated the branching ratios for the decays $\bar{B} \rightarrow D^{(*)} l \bar{\nu}$. The results obtained using $V_{c b}=0.043$ from [7] are

$$
\begin{aligned}
B R(\bar{B} \rightarrow D l \bar{\nu}) & =1.80\left(\tau_{B} / 1.29 p s\right) \% \\
B R\left(\bar{B} \rightarrow D^{*} l \bar{\nu}\right) & =5.46\left(\tau_{B} / 1.29 p s\right) \%
\end{aligned}
$$

to be comapred with the experimental results [7] (averaged over the charges of the decaying $B)(1.7 \pm 0.4) \%$ and $(4.8 \pm 0.6) \%$.

We predict moreover

$$
\begin{aligned}
B R\left(\bar{B}_{s} \rightarrow D_{s} l \bar{\nu}\right) & =1.51\left(\tau_{B_{s}} / 1.29 p s\right) \% \\
B R\left(\bar{B}_{s} \rightarrow D_{s}^{(*)} l \bar{\nu}\right) & =4.95\left(\tau_{B_{s}} / 1.29 p s\right) \% \\
B R\left(\Lambda_{b} \rightarrow \Lambda_{c} l \bar{\nu}\right) & =6.09\left(\tau_{\Lambda_{b}} / 1.29 p s\right) \%
\end{aligned}
$$

For these decays one can as yet make no comparison with experiment. Theoretical predictions, on the other hand, are ambiguous. Thus, Jenkins et al. conclude from chiral perturbation theory that the ratio $\xi^{B_{s}}(\omega) / \xi^{B}(\omega)$ should be an increasing function of $\omega[23]$. The bag model gives the opposite prediction. The bag model predicts that the function $\xi^{\Lambda}(\omega)$ decreses with increasing $\omega$ much faster than the function $\xi^{B}(\omega)$. A similar prediction is made by König et al 22 from a calculation of overlaps in the infinite momentum frame. On the other hand, models based on meson exchange [15, 21] suggest that the Isgur-Wise functions for the two cases should be similar. A 
steeper Isgur-Wise function means that the ground state to ground state semileptonic decays are a smaller fraction of all the semileptonic decays of the parent particle. Experimental data should soon shed some light on these questions.

Let us note finally that the function $\xi^{B}(\omega)$ can be also used to get a crude estimate of the branching ratio for rare decays like $B \rightarrow K^{*} \gamma$ [24]. Our function is significantly smaller than the function used in ref. 24] in the region relevant for the $b \rightarrow c$ transitions. For the $B \rightarrow K^{*}$ transition, however, which corresponds to $\omega \approx 3$, the two functions coincide. Thus we support the results from ref. 24].

\section{Conclusions}

The (modified) MIT bag model gives plausible predictions for the IsgurWise functions corresponding to the groud state to ground state transitions. Several results seem more general than the model used:

- The parameter $\rho^{2}$ should satisfy inequality (23), which is stronger than the Bjorken inequality [18.

- The Isgur-Wise functions for baryons should be much steeper functions of $\omega$ than the corresponding functions for mesons. For corresponding mesons and baryons the relation between the parameters $\rho^{2}$ is $\rho_{\text {baryon }}^{2}=$ $2 \rho_{\text {meson }}^{2}-0.25$.

- The Isgur-Wise function for the decays $\bar{B}_{s} \rightarrow D_{s}^{(*)} l \bar{\nu}$ should be somewhat steeper than those for the decays $\bar{B} \rightarrow D^{(*)} l \bar{\nu}$. With the quark mass increasing from about zero to $m_{s}$, the slight decrease of the radius pointed out in [23] is in our approach more than compensated by the increase of the quark energy.

Also the observation that the Isgur-Wise function for $\bar{B} \rightarrow D^{(*)} l \bar{\nu}$ from the present analysis is almost identical with the function obtained by Neubert [13] from sum rules, may mean that our results are more general than the model used to derive them.

The calculation is the most reliable for small recoil velocities, but we find plausible results for all recoils of interest. This is analogous to the analysis in the framework of the nonrelativistic quark model as presented in 
ref. [1]. There, however, the Isgur-Wise functions obtained seem to be too

flat. The original result $\rho^{2} \approx 0.3$ is multiplied by two in order to get a more reasonable $\omega$ dependence [1]. We explain this fact as a result of two factors: In the nonrelativistic calculation the boosts, which contribute 0.25 to $\rho^{2}$, are absent. Moreover, the quark energy $E_{q}$ is replaced by the constituent quark mass, which is smaller.

The overall agreement of our results with the little data available is good, but there are hints that we may be overestimating the branching ratio for the decay $\bar{B} \rightarrow D^{*} l \bar{\nu}$. If experiment supports this, it would be a confirmation of the analysis of Amundson and Rosner [25], who find that the $O\left(1 / m_{Q}\right)$ and the QCD corrections cancel for the decay $\bar{B} \rightarrow D l \bar{\nu}$, while the negative QCD correction dominates for $\bar{B} \rightarrow D^{*} l \bar{\nu}$.

\section{References}

[1] N. Isgur, D. Scora, B. Grinstein and M.B. Wise, Phys. Rev. D39(1989)799.

[2] N. Isgur and M.B. Wise, Phys. Letters B237(1990)527.

[3] W.A. Ponce, Phys. Rev. D19(1979)197.

[4] E.V. Shuryak, Phys. Letters B93(1980)134.

[5] D. Isatt, C. De Tar and M. Stephenson, Nucl. Phys. B199 (1982)269.

[6] M. Sadzikowski, Acta Phys. Pol. B in print.

[7] Partcle Data Group, Phys. Rev. D45(1992)Part 2.

[8] K. Zalewski, Phys. Letters B264(1991)432.

[9] N. Isgur, Phys. Rev. D43(1991)810.

[10] J. Rosner, Phys. Rev. D42(1990)3732.

[11] M. Neubert and V. Rieckert, Nucl. Phys. B282(1992)97.

[12] A. V. Radyushkin, Phys. Letters, B271(1991)218. 
[13] M. Neubert, Phys. Rev. D45(1991)2451.

[14] E. De Rafael and J. Taron, Phys. Letters B282(1992)215.

[15] T. A. Mannel and A. Schuler, Phys. Letters B279(1992)194.

[16] G. V. Efimov et al., Z. Phys. C - Particles and Fields 54(1992)349.

[17] H. Albrecht et al., (ARGUS Collaboration) "Investigation of the decays $\bar{B} \rightarrow D^{*+} l^{-} \bar{\nu}$ and $\bar{B} \rightarrow D^{* *} l^{-} \bar{\nu}$. DESY preprint $92-146$ (1992).

[18] J.D. Bjorken, in ed. M. Greco, Proceedings of the $4^{\text {th }}$ Rencontre de la Vallee d' Aoste, La Thuile, Italy 1990. Editions Frontiere, Gif sur Yvette, France (1990).

[19] Ø. Lie-Svendsen and H. Hфgaasen, Z. Phys. C - Particles and Fields C35(1987)239.

[20] M. Blok and M. Shifman, "The Isgur-Wise function in the small velocity limit" Minnesota preprint TPI-MINN-92-32/T (1992).

[21] R. Singleton Jr., Phys. Rev. D43(1991)2939.

[22] B. König, J.G. Körner. M. Krämer and P. Kroll, Mainz preprint WZTH/92-42, December (1992).

[23] E. Jenkins and M.J. Savage, Phys. Letters B281(1992)331.

[24] A. Ali, T. Ohl and T. Mannel, Phys. Letters B298(1993)195.

[25] J.E. Amundson and J.L. Rosner, "Heavy quark symmetry violation in semileptonic decays of $D$ mesons", Chicago preprint EFI-92-36 October (1992). 